\title{
Scale-up synthesis of lipase-catalyzed palm esters in stirred-tank reactor
}

\begin{abstract}
Lipase-catalyzed production of palm esters by alcoholysis of palm oil with oleyl alcohol in nhexane was performed in $2 \mathrm{~L}$ stirred-tank reactor (STR). Investigation on the performance of reactor operation was carried out in batch mode STR with single impeller mounted on the centrally located shaft. Rushton turbine (RT) impellers provide the highest reaction yield (95.8\%) at lower agitation speed as compared to AL-hydrofoil (AL-H) and 2-bladed elephant ear (EE) impellers. Homogenous enzyme particles suspension was obtained at $250 \mathrm{rpm}$ by using RT impeller. At higher impeller speed, the shear effect on the enzyme particles caused by agitation has decreased the reaction performance. Palm esters reaction mixture in STR follows Newtons' law due to the linear relation between the shear stress (s) and shear rate (dt/dy). High stability of Lipozyme RM IM was observed as shown by its ability to be repeatedly used to give high percentage yield (79\%) of palm esters even after 15 cycles of reaction. The process was successfully scale-up to $75 \mathrm{~L} \mathrm{STR} \mathrm{(50} \mathrm{L} \mathrm{working} \mathrm{volume)} \mathrm{based}$ on a constant impeller tip speed approach, which gave the yield of $97.2 \%$ after $5 \mathrm{~h}$ reaction time.
\end{abstract}

Keyword: Palm esters, Stirred-tank reactor, Single impeller, Enzyme suspension, Operational stability 